\title{
Formalization of the MRDP Theorem in the Mizar System ${ }^{11}$
}

\author{
Karol Pąk \\ Institute of Informatics \\ University of Białystok \\ Poland
}

\begin{abstract}
Summary. This article is the final step of our attempts to formalize the negative solution of Hilbert's tenth problem.

In our approach, we work with the Pell's Equation defined in 2]. We analyzed this equation in the general case to show its solvability as well as the cardinality and shape of all possible solutions. Then we focus on a special case of the equation, which has the form $x^{2}-\left(a^{2}-1\right) y^{2}=1[8]$ and its solutions considered as two sequences $\left\{x_{i}(a)\right\}_{i=0}^{\infty},\left\{y_{i}(a)\right\}_{i=0}^{\infty}$. We showed in [1] that the $n$-th element of these sequences can be obtained from lists of several basic Diophantine relations as linear equations, finite products, congruences and inequalities, or more precisely that the equation $x=y_{i}(a)$ is Diophantine. Following the post-Matiyasevich results we show that the equality determined by the value of the power function $y=x^{z}$ is Diophantine, and analogously property in cases of the binomial coefficient, factorial and several product 9 .

In this article, we combine analyzed so far Diophantine relation using conjunctions, alternatives as well as substitution to prove the bounded quantifier theorem. Based on this theorem we prove MDPR-theorem that every recursively enumerable set is Diophantine, where recursively enumerable sets have been defined by the Martin Davis normal form.

The formalization by means of Mizar system [5], 7], 4] follows [10, Z. Adamowicz, P. Zbierski [3] as well as M. Davis [6].
\end{abstract}

\section{MSC: 11D45 68T99 03B35}

Keywords: Hilbert's 10th problem; Diophantine relations

MML identifier: HILB10_5, version: 8.1.09 5.57.1355

\footnotetext{
${ }^{1}$ This work has been financed by the resources of the Polish National Science Centre granted by decision no. DEC-2015/19/D/ST6/01473. 


\section{Preliminaries}

From now on $i, j, n, n_{1}, n_{2}, m, k, l, u$ denote natural numbers, $i_{1}, i_{2}, i_{3}, i_{4}$, $i_{5}, i_{6}$ denote elements of $n, p, q$ denote $n$-element finite 0 -sequences of $\mathbb{N}$, and $a, b, c, d, e, f$ denote integers.

Let $n$ be a natural number. Let us note that idseq $(n)$ is $\mathbb{Z}$ valued.

Let $x$ be an $n$-element, natural-valued finite 0 -sequence and $p$ be a $\mathbb{Z}$ valued polynomial of $n, \mathbb{R}_{\mathrm{F}}$. One can check that $\operatorname{eval}\left(p,{ }^{\circledR} x\right)$ is integer.

Now we state the proposition:

(1) Let us consider a $\mathbb{Z}$ valued polynomial $p$ of $n, \mathbb{R}_{\mathrm{F}}$, and $n$-element finite 0 -sequences $x, y$ of $\mathbb{N}$. Suppose $k \neq 0$ and for every $i$ such that $i \in n$ holds $k \mid x(i)-y(i)$. Then $k \mid\left(\operatorname{eval}\left(p,{ }^{\circledR} x\right)\right.$ qua integer $)-\left(\operatorname{eval}\left(p,{ }^{\circledR} y\right)\right.$ qua integer $)$. ProOF: Reconsider $f_{1}=\mathbb{R}_{\mathrm{F}}$ as a field. Reconsider $p_{1}=p$ as a polynomial of $n, f_{1}$. Reconsider $x_{2}={ }^{\circledR} x, y_{2}={ }^{\circledR} y$ as a function from $n$ into the carrier of $f_{1}$. Set $s_{3}=\operatorname{SgmX}\left(\operatorname{BagOrder} n\right.$, Support $\left.p_{1}\right)$. Consider $X$ being a finite sequence of elements of the carrier of $f_{1}$ such that len $X=$ len $s_{3}$ and $\operatorname{eval}\left(p_{1}, x_{2}\right)=\sum X$ and for every element $i$ of $\mathbb{N}$ such that $1 \leqslant i \leqslant \operatorname{len} X$ holds $X_{/ i}=p_{1} \cdot s_{3 / i} \cdot\left(\operatorname{eval}\left(s_{3 / i}, x_{2}\right)\right)$.

Consider $Y$ being a finite sequence of elements of the carrier of $f_{1}$ such that len $Y=$ len $s_{3}$ and $\operatorname{eval}\left(p_{1}, y_{2}\right)=\sum Y$ and for every element $i$ of $\mathbb{N}$ such that $1 \leqslant i \leqslant \operatorname{len} Y$ holds $Y_{/ i}=p_{1} \cdot s_{3 / i} \cdot\left(\operatorname{eval}\left(s_{3 / i}, y_{2}\right)\right)$. Reconsider $Y_{2}=Y, X_{4}=X$ as a finite sequence of elements of $\mathbb{R}$. Define $\mathcal{P}$ [natural number] $\equiv$ if $\$_{1} \leqslant$ len $X$, then $\sum\left(X_{4} \mid \$_{1}\right)-\sum\left(Y_{2} \mid \$_{1}\right)$ is an integer and for every integer $d$ such that $d=\sum\left(X_{4} \uparrow \$_{1}\right)-\sum\left(Y_{2} \mid \$_{1}\right)$ holds $k \mid d$. For every natural number $i$ such that $\mathcal{P}[i]$ holds $\mathcal{P}[i+1]$. $\mathcal{P}[i]$.

Let $f$ be a $\mathbb{Z}$ valued function. Let us note that $-f$ is $\mathbb{Z}$ valued.

The scheme $S C H 1$ deals with a binary predicate $\mathcal{P}$ and a finite-0-sequenceyielding finite 0 -sequence $f$ and states that

(Sch. 1) $\{f(i)(j)$, where $i, j$ are natural numbers $: \mathcal{P}[i, j]\}$ is finite.

Now we state the propositions:

(2) If $m \geqslant n>0$, then $1+m$ ! $\cdot(\operatorname{idseq}(n))$ is a CR-sequence.

Proof: Set $h=1+m ! \cdot(\operatorname{idseq}(n))$. Define $\mathcal{F}$ (natural number) $=m ! \cdot \$_{1}+1$. For every $i$ such that $i \in \operatorname{dom} h$ holds $h(i)=\mathcal{F}(i)$. $h$ is positive yielding. For every natural numbers $i, j$ such that $i, j \in \operatorname{dom} h$ and $i<j$ holds $h(i)$ and $h(j)$ are relatively prime. $h$ is Chinese remainder.

(3) Let us consider a prime number $p$, and a finite sequence $f$ of elements of $\mathbb{N}$. Suppose $f$ is positive yielding and $p \mid \prod f$. Then there exists $i$ such that

(i) $i \in \operatorname{dom} f$, and 
(ii) $p \mid f(i)$.

Proof: Define $\mathcal{P}$ [natural number] $\equiv$ for every finite sequence $f$ of elements of $\mathbb{N}$ such that len $f=\$_{1}$ and $f$ is positive yielding and $p \mid \prod f$ there exists $i$ such that $i \in \operatorname{dom} f$ and $p \mid f(i)$. $\mathcal{P}[0]$. If $\mathcal{P}[n]$, then $\mathcal{P}[n+1] . \mathcal{P}[n]$.

\section{Selected Operations on Polynomials}

Let $n$ be a set and $p$ be a series of $n, \mathbb{R}_{\mathrm{F}}$. The functor $|p|$ yielding a series of $n, \mathbb{R}_{\mathrm{F}}$ is defined by

(Def. 1) for every bag $b$ of $n, i t(b)=|p(b)|$.

Now we state the proposition:

(4) Let us consider a set $n$, and a series $p$ of $n, \mathbb{R}_{\mathrm{F}}$. Then $\operatorname{Support} p=$ Support $|p|$.

Let $n$ be an ordinal number and $p$ be a polynomial of $n, \mathbb{R}_{\mathrm{F}}$. Let us note that $|p|$ is finite-Support.

Let $n$ be a set, $S$ be a non empty zero structure, and $p$ be a finite-Support series of $n, S$. One can check that Support $p$ is finite.

Let $n$ be an ordinal number, $L$ be an add-associative, right zeroed, right complementable, non empty additive loop structure, and $p$ be a polynomial of $n, L$. The functor $\sum \operatorname{coeff}(p)$ yielding an element of $L$ is defined by the term

(Def. 2) $\sum p \cdot(\operatorname{SgmX}(\operatorname{BagOrder} n, \operatorname{Support} p))$.

The functor degree $(p)$ yielding a natural number is defined by

(Def. 3) (i) there exists a bag $s$ of $n$ such that $s \in \operatorname{Support} p$ and $i t=\operatorname{degree}(s)$ and for every bag $s_{1}$ of $n$ such that $s_{1} \in \operatorname{Support} p$ holds $\operatorname{degree}\left(s_{1}\right) \leqslant$ $i t$, if $p \neq 0_{n} L$,

(ii) it $=0$, otherwise.

Now we state the propositions:

(5) Let us consider an ordinal number $n$, and a bag $b$ of $n$. Then $\operatorname{degree}(b)=$ $\sum b \cdot\left(\operatorname{SgmX}\left(\subseteq_{n}, \operatorname{support} b\right)\right)$.

(6) Let us consider an ordinal number $n$, an add-associative, right zeroed, right complementable, non empty additive loop structure $L$, and a polynomial $p$ of $n, L$. Then $\operatorname{degree}(p)=0$ if and only if Support $p \subseteq$ $\{$ EmptyBag $n\}$.

Proof: If degree $(p)=0$, then Support $p \subseteq\{\operatorname{EmptyBag} n\}$. Consider $s$ being a bag of $n$ such that $s \in \operatorname{Support} p$ and degree $(p)=\operatorname{degree}(s)$.

(7) Let us consider an ordinal number $n$, an add-associative, right zeroed, right complementable, non empty additive loop structure $L$, a polynomial $p$ of $n, L$, and a bag $b$ of $n$. If $b \in \operatorname{Support} p$, then $\operatorname{degree}(p) \geqslant \operatorname{degree}(b)$. 
(8) Let us consider an ordinal number $n$, and a polynomial $p$ of $n, \mathbb{R}_{\mathrm{F}}$. If $|p|=0_{n}\left(\mathbb{R}_{\mathrm{F}}\right)$, then $p=0_{n}\left(\mathbb{R}_{\mathrm{F}}\right)$.

Let $n$ be a set. One can verify that $\left|0_{n}\left(\mathbb{R}_{\mathrm{F}}\right)\right|$ reduces to $0_{n}\left(\mathbb{R}_{\mathrm{F}}\right)$. Now we state the propositions:

(9) Let us consider an ordinal number $n$, and a polynomial $p$ of $n, \mathbb{R}_{\mathrm{F}}$. Then $\operatorname{degree}(p)=\operatorname{degree}(|p|)$. The theorem is a consequence of (8) and (4).

(10) Let us consider an ordinal number $n$, a bag $b$ of $n$, and a real number $r$. Suppose $r \geqslant 1$. Let us consider a function $x$ from $n$ into the carrier of $\mathbb{R}_{\mathrm{F}}$. Suppose for every object $i$ such that $i \in \operatorname{dom} x$ holds $|x(i)| \leqslant r$. Then $|\operatorname{eval}(b, x)| \leqslant r^{\text {degree }(b)}$.

Proof: Reconsider $f_{1}=\mathbb{R}_{\mathrm{F}}$ as a field. Set $s_{2}=\operatorname{SgmX}\left(\subseteq_{n}\right.$, support $\left.b\right)$. Set $B=b \cdot s_{2}$. Consider $y$ being a finite sequence of elements of $f_{1}$ such that len $y=$ len $s_{2}$ and $\operatorname{eval}(b, x)=\prod y$ and for every element $i$ of $\mathbb{N}$ such that $1 \leqslant i \leqslant$ len $y$ holds $y_{/ i}=\operatorname{power}_{\mathbb{R}_{\mathrm{F}}}\left(x \cdot s_{2 / i}, B_{/ i}\right)$.

Define $\mathcal{P}$ [natural number] $\equiv$ if $\$_{1} \leqslant$ len $y$, then $\prod\left(y\left\lceil \$_{1}\right)\right.$ is a real number and for every real number $P$ such that $P=\prod\left(y\left\lceil \$_{1}\right)\right.$ holds $|P| \leqslant$ $r^{\sum\left(B \$_{1}\right)}$. For every $i$ such that $\mathcal{P}[i]$ holds $\mathcal{P}[i+1]$. For every $i, \mathcal{P}[i]$.

(11) Let us consider an ordinal number $n$, a polynomial $p$ of $n, \mathbb{R}_{\mathrm{F}}$, and a real number $r$. Suppose $r \geqslant 1$. Let us consider a function $x$ from $n$ into the carrier of $\mathbb{R}_{\mathrm{F}}$. Suppose for every object $i$ such that $i \in \operatorname{dom} x \operatorname{holds}|x(i)| \leqslant r$. Then $|\operatorname{eval}(p, x)| \leqslant\left(\sum \operatorname{coeff}(|p|)\right) \cdot\left(r^{\text {degree }(p)}\right)$.

PROOF: Reconsider $f_{1}=\mathbb{R}_{\mathrm{F}}$ as a field. Reconsider $p_{1}=p, A_{1}=|p|$ as a polynomial of $n, f_{1}$. Reconsider $x_{2}=x$ as a function from $n$ into the carrier of $f_{1}$. Set $S_{1}=\operatorname{SgmX}\left(\operatorname{BagOrder} n\right.$, Support $\left.p_{1}\right)$. Reconsider $H=A_{1} \cdot S_{1}$ as a finite sequence of elements of the carrier of $\mathbb{R}_{\mathrm{F}} \cdot \sum \operatorname{coeff}(|p|)=\sum A_{1} \cdot S_{1}$.

Consider $y$ being a finite sequence of elements of the carrier of $f_{1}$ such that len $y=\operatorname{len} S_{1}$ and $\operatorname{eval}(p, x)=\sum y$ and for every element $i$ of $\mathbb{N}$ such that $1 \leqslant i \leqslant$ len $y$ holds $y_{/ i}=p_{1} \cdot S_{1 / i} \cdot\left(\operatorname{eval}\left(S_{1 / i}, x_{2}\right)\right)$. Reconsider $Y=y$ as a finite sequence of elements of $\mathbb{R}$. Define $\mathcal{P}$ [natural number] $\equiv$ if $\$_{1} \leqslant$ len $y$, then $\mid \sum\left(Y\left\lceil \$_{1}\right) \mid \leqslant\left(\sum\left(H\left\lceil \$_{1}\right)\right) \cdot\left(r^{\text {degree }(p)}\right)\right.\right.$. For every natural number $i$ such that $\mathcal{P}[i]$ holds $\mathcal{P}[i+1]$. For every natural number $i, \mathcal{P}[i]$.

Let $n$ be an ordinal number and $p$ be a $\mathbb{Z}$ valued polynomial of $n, \mathbb{R}_{\mathrm{F}}$. Let us note that $|p|$ is natural-valued and there exists a polynomial of $n, \mathbb{R}_{\mathrm{F}}$ which is natural-valued.

Let $O$ be an ordinal number and $p$ be a natural-valued polynomial of $O, \mathbb{R}_{\mathrm{F}}$. Let us observe that $\sum \operatorname{coeff}(p)$ is natural. 


\section{Selected Subsets of Zero Based Finite Sequences of $\mathbb{N}$ as Diophantine Sets}

The scheme SubsetDioph deals with a natural number $n$ and a 4 -ary predicate $\mathcal{P}$ and a set $\mathcal{S}$ and states that

(Sch. 2) For every elements $i_{2}, i_{3}, i_{4}$ of $n,\{p$, where $p$ is an $n$-element finite 0 -sequence of $\mathbb{N}$ : for every natural number $i$ such that $i \in \mathcal{S}$ holds $\left.\mathcal{P}\left[p(i), p\left(i_{2}\right), p\left(i_{3}\right), p\left(i_{4}\right)\right]\right\}$ is a Diophantine subset of the $n$-xtuples of $\mathbb{N}$ provided

- for every elements $i_{1}, i_{2}, i_{3}, i_{4}$ of $n,\{p$, where $p$ is an $n$-element finite 0 -sequence of $\left.\mathbb{N}: \mathcal{P}\left[p\left(i_{1}\right), p\left(i_{2}\right), p\left(i_{3}\right), p\left(i_{4}\right)\right]\right\}$ is a Diophantine subset of the $n$-xtuples of $\mathbb{N}$ and

- $\mathcal{S} \subseteq \mathbb{Z}_{n}$.

Now we state the propositions:

(12) Suppose $n_{1}+n_{2} \leqslant n$.

Then $\left\{p: p\left(i_{1}\right) \geqslant k \cdot\left(\left(p\left(i_{2}\right)^{2}+1\right) \cdot\left(\prod\left(1+p_{\left\lfloor n_{1}\right.}\left\lceil n_{2}\right)\right) \cdot\left(l \cdot p\left(i_{3}\right)+m\right)^{i \cdot p\left(i_{4}\right)+j}\right)\right\}\right.$ is a Diophantine subset of the $n$-xtuples of $\mathbb{N}$.

PROOF: Define $\mathcal{F}_{0}$ (natural number, natural number, natural number) $=$ $\$_{1}{ }^{\$_{2}}$. Define $\mathcal{P}_{0}$ [natural number, natural number, natural object, natural number, natural number, natural number $] \equiv 1 \cdot \$_{1} \geqslant k \cdot \$_{3}+0$. For every $i_{1}$, $i_{2}, i_{3}, i_{4}$, and $i_{5},\left\{p: \mathcal{P}_{0}\left[p\left(i_{1}\right), p\left(i_{2}\right), \mathcal{F}_{0}\left(p\left(i_{3}\right), p\left(i_{4}\right), p\left(i_{5}\right)\right), p\left(i_{3}\right), p\left(i_{4}\right), p\left(i_{5}\right)\right]\right\}$ is a Diophantine subset of the $n$-xtuples of $\mathbb{N}$. Define $\mathcal{F}_{1}$ (natural number, natural number, natural number $)=i \cdot \$_{1}+j$. Define $\mathcal{P}_{1}$ [natural number, natural number, natural object, natural number, natural number, natural number $] \equiv \$_{1} \geqslant k \cdot\left(\$_{2}{ }^{\$_{3}}\right)$. For every $i_{1}, i_{2}, i_{3}, i_{4}$, and $i_{5},\left\{p: \mathcal{P}_{1}\left[p\left(i_{1}\right), p\left(i_{2}\right), \mathcal{F}_{1}(p\right.\right.$ $\left.\left.\left.\left(i_{3}\right), p\left(i_{4}\right), p\left(i_{5}\right)\right), p\left(i_{3}\right), p\left(i_{4}\right), p\left(i_{5}\right)\right]\right\}$ is a Diophantine subset of the $n$-xtuples of $\mathbb{N}$. Define $\mathcal{F}_{2}$ (natural number, natural number, natural number) $=1 \cdot \$_{1}$. $\$ 2$.

Define $\mathcal{P}_{2}$ [natural number, natural number, natural object, natural number, natural number, natural number $] \equiv \$_{1} \geqslant k \cdot\left(\$_{3}{ }^{i \cdot \$_{2}+j}\right)$. For every $i_{1}$, $i_{2}, i_{3}, i_{4}$, and $i_{5},\left\{p: \mathcal{P}_{2}\left[p\left(i_{1}\right), p\left(i_{2}\right), \mathcal{F}_{2}\left(p\left(i_{3}\right), p\left(i_{4}\right), p\left(i_{5}\right)\right), p\left(i_{3}\right), p\left(i_{4}\right), p\left(i_{5}\right)\right]\right\}$ is a Diophantine subset of the $n$-xtuples of $\mathbb{N}$. Define $\mathcal{P}_{3}$ [natural number, natural number, natural object, natural number, natural number, natural number $] \equiv \$_{1} \geqslant k \cdot\left(\$_{6} \cdot \$_{3} \cdot \$_{2}+j\right)$. For every $i_{1}, i_{2}, i_{3}, i_{4}$, and $i_{5},\{p$ $\left.: \mathcal{P}_{3}\left[p\left(i_{1}\right), p\left(i_{2}\right), \mathcal{F}_{2}\left(p\left(i_{3}\right), p\left(i_{4}\right), p\left(i_{5}\right)\right), p\left(i_{3}\right), p\left(i_{4}\right), p\left(i_{5}\right)\right]\right\}$ is a Diophantine subset of the $n$-xtuples of $\mathbb{N}$. Define $\mathcal{F}_{5}$ (natural number, natural number, natural number $)=1 \cdot \$_{1}+1$. Define $\mathcal{P}_{5}$ [natural number, natural number, natural object, natural number, natural number, natural number $] \equiv \$_{1} \geqslant k$. $\left(\$_{3} \cdot \$_{5} \cdot \$_{6} \cdot \$_{2}+j\right)$. For every $i_{1}, i_{2}, i_{3}, i_{4}$, and $i_{5},\left\{p: \mathcal{P}_{5}\left[p\left(i_{1}\right), p\left(i_{2}\right), \mathcal{F}_{5}\left(p\left(i_{3}\right)\right.\right.\right.$, 
$\left.\left.\left.p\left(i_{4}\right), p\left(i_{5}\right)\right), p\left(i_{3}\right), p\left(i_{4}\right), p\left(i_{5}\right)\right]\right\}$ is a Diophantine subset of the $n$-xtuples of $\mathbb{N}$. Define $\mathcal{G}$ (natural number, natural number, natural number) $=l$. $\$_{1}+m$. Define $\mathcal{R}_{1}$ [natural number, natural number, natural object, natural number, natural number, natural number $] \equiv \$_{1} \geqslant k \cdot\left(\$_{3} \cdot \$_{5} \cdot\left(\$_{6}+1\right)^{i \cdot \$_{2}+j}\right)$. For every $i_{1}, i_{2}, i_{3}, i_{4}$, and $i_{5},\left\{p: \mathcal{R}_{1}\left[p\left(i_{1}\right), p\left(i_{2}\right), \mathcal{G}\left(p\left(i_{3}\right), p\left(i_{4}\right), p\left(i_{5}\right)\right), p\left(i_{3}\right)\right.\right.$, $\left.\left.p\left(i_{4}\right), p\left(i_{5}\right)\right]\right\}$ is a Diophantine subset of the $n$-xtuples of $\mathbb{N}$.

Define $\mathcal{P}_{6}$ [natural number, natural number, natural object, natural number, natural number, natural number $] \equiv \$_{1} \geqslant k \cdot\left(\left(\$_{3}+1\right) \cdot \$_{5} \cdot\left(l \cdot \$_{6}+\right.\right.$ $m)^{i \cdot \$_{2}+j}$. Define $\mathcal{F}_{6}$ (natural number, natural number, natural number) $=$ $1 \cdot \$_{1} \cdot \$_{1}$. For every $n, i_{1}, i_{2}, i_{3}, i_{4}$, and $i_{5},\left\{p: \mathcal{P}_{6}\left[p\left(i_{1}\right), p\left(i_{2}\right), \mathcal{F}_{6}\left(p\left(i_{3}\right), p\left(i_{4}\right)\right.\right.\right.$, $\left.\left.\left.p\left(i_{5}\right)\right), p\left(i_{3}\right), p\left(i_{4}\right), p\left(i_{5}\right)\right]\right\}$ is a Diophantine subset of the $n$-xtuples of $\mathbb{N}$. Set $X=n+1$. Reconsider $N=n, I_{1}=i_{1}, I_{2}=i_{2}, I_{3}=i_{3}, I_{4}=i_{4}$ as an element of $X$. Define $\mathcal{P}_{7}$ [finite 0 -sequence of $\left.\mathbb{N}\right] \equiv \$_{1}\left(I_{1}\right) \geqslant k$. $\left(\left(1 \cdot \$_{1}\left(I_{2}\right) \cdot \$_{1}\left(I_{2}\right)+1\right) \cdot \$_{1}(N) \cdot\left(l \cdot \$_{1}\left(I_{3}\right)+m\right)^{i \cdot \$_{1}\left(I_{4}\right)+j}\right)$. Define $\mathcal{Q}_{7}$ [finite 0 -sequence of $\mathbb{N}] \equiv \$_{1}(N)=\prod\left(1+\$_{1\left\lfloor n_{1}\right.}\left\lceil n_{2}\right)\right.$. Set $P_{1}=\{p$, where $p$ is an $X$-element finite 0 -sequence of $\mathbb{N}: \mathcal{P}_{7}[p]$ and $\left.\mathcal{Q}_{7}[p]\right\}$. $P_{1}$ is a Diophantine subset of the $X$-xtuples of $\mathbb{N}$. Define $\mathcal{S}$ [finite 0 -sequence of $\mathbb{N}] \equiv$ $\$_{1}\left(i_{1}\right) \geqslant k \cdot\left(\left(\$_{1}\left(i_{2}\right)^{2}+1\right) \cdot\left(\prod\left(1+\$_{1\left\lfloor n_{1}\right.}\left\lceil n_{2}\right)\right) \cdot\left(l \cdot \$_{1}\left(i_{3}\right)+m\right)^{i \cdot \$_{1}\left(i_{4}\right)+j}\right)\right.$. Set $S=\{p: \mathcal{S}[p]\} . S \subseteq$ the $n$-xtuples of $\mathbb{N}$.

(13) Let us consider a $\mathbb{Z}$ valued polynomial $P$ of $k, \mathbb{R}_{\mathrm{F}}$, an integer $a$, a permutation $p_{2}$ of $n$, and $i_{1}$. Suppose $k \leqslant n$. Then $\{p$ : for every $k$-element finite 0 -sequence $q$ of $\mathbb{N}$ such that $q=p \cdot p_{2}\left\lceil k\right.$ holds $\left.a \cdot p\left(i_{1}\right)=\operatorname{eval}\left(P,{ }^{\circledR} q\right)\right\}$ is a Diophantine subset of the $n$-xtuples of $\mathbb{N}$.

(14) Let us consider a $\mathbb{Z}$ valued polynomial $P$ of $k+1, \mathbb{R}_{\mathrm{F}}$, an integer $a, n, i_{1}$, and $i_{2}$. Suppose $k+1 \leqslant n$ and $k \in i_{2}$. Then $\{p$ : for every $(k+1)$-element finite 0-sequence $q$ of $\mathbb{N}$ such that $q=\left\langle p\left(i_{2}\right)\right\rangle^{\frown}\left(p\lceil k)\right.$ holds $a \cdot p\left(i_{1}\right)=$ $\left.\operatorname{eval}\left(P,{ }^{@} q\right)\right\}$ is a Diophantine subset of the $n$-xtuples of $\mathbb{N}$.

Proof: Set $k_{1}=k+1$. Reconsider $I_{5}=\mathrm{id}_{k}$ as a finite 0 -sequence. Set $f=\left\langle i_{2}\right\rangle^{\frown} I_{5}$. Set $R=\operatorname{rng} f$. Consider $g$ being a function such that $g$ is one-to-one and $\operatorname{dom} g=n \backslash k_{1}$ and $\operatorname{rng} g=n \backslash R$. Reconsider $f_{1}=$ $f+\cdot g$ as a function from $n$ into $n$. Define $\mathcal{Q}[$ finite 0 -sequence of $\mathbb{N}] \equiv$ for every $k_{1}$-element finite 0 -sequence $q$ of $\mathbb{N}$ such that $q=\$_{1} \cdot f_{1} \backslash k_{1}$ holds $a \cdot \$_{1}\left(i_{1}\right)=\operatorname{eval}\left(P,{ }^{\circledR} q\right)$. Define $\mathcal{R}$ [finite 0 -sequence of $\left.\mathbb{N}\right] \equiv$ for every $(k+1)$-element finite 0 -sequence $q$ of $\mathbb{N}$ such that $q=\left\langle \$_{1}\left(i_{2}\right)\right\rangle \frown\left(\$_{1}\lceil k)\right.$ holds $a \cdot \$_{1}\left(i_{1}\right)=\operatorname{eval}\left(P,{ }^{@} q\right)$. For every $n$-element finite 0 -sequence $p$ of $\mathbb{N}$, $\mathcal{Q}[p]$ iff $\mathcal{R}[p] .\{p: \mathcal{Q}[p]\}=\{q: \mathcal{R}[q]\}$.

(15) Let us consider a $\mathbb{Z}$ valued polynomial $P$ of $k+1, \mathbb{R}_{\mathrm{F}}, n, i_{1}$, and $i_{2}$. Suppose $k+1 \leqslant n$ and $k \in i_{1}$. Then $\{p$ : for every $(k+1)$-element finite 0 -sequence $q$ of $\mathbb{N}$ such that $q=\left\langle p\left(i_{1}\right)\right\rangle^{\frown}\left(p\lceil k) \operatorname{holds} \operatorname{eval}\left(P,{ }^{\circledR} q\right) \equiv 0\left(\bmod p\left(i_{2}\right)\right)\right\}$ is 
a Diophantine subset of the $n$-xtuples of $\mathbb{N}$.

Proof: Set $k_{1}=k+1$. Set $X=n+1$. Reconsider $N=n, I_{1}=i_{1}$, $I_{2}=i_{2}$ as an element of $X$. Define $\mathcal{P}$ [finite 0 -sequence of $\left.\mathbb{N}\right] \equiv 1 \cdot \$_{1}(N) \equiv$ $0 \cdot \$_{1}\left(I_{1}\right)\left(\bmod 1 \cdot \$_{1}\left(I_{2}\right)\right)$. Define $\mathcal{O}$ [finite 0 -sequence of $\left.\mathbb{N}\right] \equiv$ for every $k_{1}$-element finite 0 -sequence $q$ of $\mathbb{N}$ such that $q=\left\langle \$_{1}\left(I_{1}\right)\right\rangle \frown\left(\$_{1}\lceil k)\right.$ holds $1 \cdot \$_{1}(N)=\operatorname{eval}\left(P,{ }^{@} q\right)$. Define $\mathcal{M}[$ finite 0 -sequence of $\mathbb{N}] \equiv$ for every $k_{1^{-}}$ element finite 0 -sequence $q$ of $\mathbb{N}$ such that $q=\left\langle \$_{1}\left(I_{1}\right)\right\rangle^{\frown}\left(\$_{1}\lceil k)\right.$ holds $(-1)$. $\$_{1}(N)=\operatorname{eval}\left(P,{ }^{@} q\right)$. Define $\mathcal{Q}[$ finite 0 -sequence of $\mathbb{N}] \equiv \mathcal{O}\left[\$_{1}\right]$ or $\mathcal{M}\left[\$_{1}\right]$. $\{p$, where $p$ is an $X$-element finite 0 -sequence of $\mathbb{N}: \mathcal{O}[p]\}$ is a Diophantine subset of the $X$-xtuples of $\mathbb{N}$. $\{p$, where $p$ is an $X$-element finite 0 -sequence of $\mathbb{N}: \mathcal{M}[p]\}$ is a Diophantine subset of the $X$-xtuples of $\mathbb{N}$. $\{p$, where $p$ is an $X$-element finite 0 -sequence of $\mathbb{N}: \mathcal{O}[p]$ or $\mathcal{M}[p]\}$ is a Diophantine subset of the $X$-xtuples of $\mathbb{N}$. Set $P_{1}=\{p$, where $p$ is an $X$-element finite 0 -sequence of $\mathbb{N}: \mathcal{P}[p]$ and $\mathcal{Q}[p]\} . P_{1}$ is a Diophantine subset of the $X$ xtuples of $\mathbb{N}$.

Set $P_{2}=\{p\lceil n$, where $p$ is an $X$-element finite 0 -sequence of $\mathbb{N}: p \in$ $\left.P_{1}\right\}$. Define $\mathcal{S}$ [finite 0 -sequence of $\left.\mathbb{N}\right] \equiv$ for every $k_{1}$-element finite 0 sequence $q$ of $\mathbb{N}$ such that $q=\left\langle \$_{1}\left(i_{1}\right)\right\rangle \frown\left(\$_{1}\lceil k)\right.$ holds eval $\left(P,{ }^{@} q\right) \equiv 0\left(\bmod \$_{1}\right.$ $\left.\left(i_{2}\right)\right)$. Set $S=\{p: \mathcal{S}[p]\} . S \subseteq P_{2} . P_{2} \subseteq S$.

\section{Bounded Quantifier Theorem And its Variant}

Let us consider a $\mathbb{Z}$ valued polynomial $p$ of $2+n+k, \mathbb{R}_{\mathrm{F}}$, an $n$-element finite 0 -sequence $X$ of $\mathbb{N}$, and an element $x$ of $\mathbb{N}$. Now we state the propositions:

(16) For every element $z$ of $\mathbb{N}$ such that $z \leqslant x$ there exists a $k$-element finite 0 -sequence $y$ of $\mathbb{N}$ such that $\operatorname{eval}\left(p,{ }^{\circledR}((\langle z, x\rangle \frown X) \frown y)\right)=0$ if and only if there exists a $k$-element finite 0 -sequence $Y$ of $\mathbb{N}$ and there exist elements $Z, e, K$ of $\mathbb{N}$ such that $K>x$ and $K \geqslant\left(\sum \operatorname{coeff}(|p|)\right)$. $\left(\left(x^{2}+1\right) \cdot(\Pi(1+X)) \cdot e^{\operatorname{degree}(p)}\right)$ and for every natural number $i$ such that $i \in k$ holds $Y(i)>e$ and $e>x$ and $1+(Z+1) \cdot(K !)=\prod(1+K ! \cdot(\operatorname{idseq}(x+$ $1)))$ and $\operatorname{eval}\left(p,{ }^{@}((\langle Z, x\rangle \frown X) \frown Y)\right) \equiv 0(\bmod 1+(Z+1) \cdot(K !))$ and for every natural number $i$ such that $i \in k$ holds $\prod(Y(i)+1+-\operatorname{idseq}(e)) \equiv$ $0(\bmod 1+(Z+1) \cdot(K !))$.

PRoOF: If for every element $z$ of $\mathbb{N}$ such that $z \leqslant x$ there exists a $k$ element finite 0 -sequence $y$ of $\mathbb{N}$ such that $\operatorname{eval}\left(p,{ }^{\circledR}((\langle z, x\rangle \frown X) \frown y)\right)=0$, then there exists a $k$-element finite 0 -sequence $Y$ of $\mathbb{N}$ and there exist elements $Z, e, K$ of $\mathbb{N}$ such that $K>x$ and $K \geqslant\left(\sum \operatorname{coeff}(|p|)\right)$. $\left(\left(x^{2}+1\right) \cdot(\Pi(1+X)) \cdot e^{\operatorname{degree}(p)}\right)$ and for every natural number $i$ such that $i \in k$ holds $Y(i)>e$ and $e>x$ and $1+(Z+1) \cdot(K !)=\prod(1+K ! \cdot(\operatorname{idseq}(x+$ 
1))) and $\left.\operatorname{eval}\left(p,{ }^{\circledR}\left((\langle Z, x\rangle\rangle^{\frown} X\right)^{\frown} Y\right)\right) \equiv 0(\bmod 1+(Z+1) \cdot(K !))$ and for every natural number $i$ such that $i \in k$ holds $\prod(Y(i)+1+-$ idseq $(e)) \equiv$ $0(\bmod 1+(Z+1) \cdot(K !))$. Set $K_{1}=K !$. Set $z_{1}=1+(z+1) \cdot K_{1}$. Consider $p_{3}$ being an element of $\mathbb{N}$ such that $p_{3} \mid z_{1}$ and $p_{3} \leqslant z_{1}$ and $p_{3}$ is prime. Define $\mathcal{P}($ object $)=Y\left(\$_{1}\right) \bmod p_{3}$.

Consider $Y_{3}$ being a finite 0 -sequence such that len $Y_{3}=k$ and for every natural number $i$ such that $i \in k$ holds $Y_{3}(i)=\mathcal{P}(i) \cdot \operatorname{rng} Y_{3} \subseteq \mathbb{N}$. Reconsider $\left.E_{1}=\operatorname{eval}\left(p,{ }^{\circledR}\left((\langle Z, x\rangle\rangle^{\frown} X\right)^{\frown} Y\right)\right)$ as an integer. $K<p_{3}$. For every $i$ such that $i \in 2+k+n$ holds $p_{3} \mid\left(\left(\langle Z, x\rangle^{\wedge} X\right)^{\wedge} Y\right)(i)-\left(\left(\langle z, x\rangle^{\frown} X\right)^{\frown}\right.$ $\left.Y_{3}\right)(i) \cdot p_{3} \mid E_{1}-\operatorname{eval}\left(p,{ }^{\circledR}\left((\langle z, x\rangle \frown X)^{\frown} Y_{3}\right)\right)$. Consider $m$ being a natural number such that $\left.\mid \operatorname{eval}\left(p,{ }^{@}((\langle z, x\rangle\urcorner X) \frown Y_{3}\right)\right) \mid=p_{3} \cdot m$. For every object $i$ such that $i \in \operatorname{dom}\left({ }^{\circledR}\left((\langle z, x\rangle \frown X) \frown Y_{3}\right)\right)$ holds $\left|\left({ }^{\circledR}\left((\langle z, x\rangle \frown X) \frown Y_{3}\right)\right)(i)\right| \leqslant$ $\left(x^{2}+1\right) \cdot(\Pi(1+X)) \cdot e \cdot\left|\operatorname{eval}\left(p,{ }^{@}\left((\langle z, x\rangle \frown X) \frown Y_{3}\right)\right)\right| \leqslant\left(\sum \operatorname{coeff}(|p|)\right) \cdot$ $\left(\left(x^{2}+1\right) \cdot\left(\prod(1+X)\right) \cdot e^{\operatorname{degree}(p)}\right)$.

(17) For every element $z$ of $\mathbb{N}$ such that $z \leqslant x$ there exists a $k$-element finite 0 -sequence $y$ of $\mathbb{N}$ such that for every $i$ such that $i \in k$ holds $y(i) \leqslant$ $x$ and $\left.\operatorname{eval}\left(p,{ }^{\circledR}((\langle z, x\rangle\urcorner X)^{\frown} y\right)\right)=0$ if and only if there exists a $k$ element finite 0 -sequence $Y$ of $\mathbb{N}$ and there exist elements $Z, K$ of $\mathbb{N}$ such that $K>x$ and $K \geqslant\left(\sum \operatorname{coeff}(|p|)\right) \cdot\left(\left(x^{2}+1\right) \cdot\left(\prod(1+X)\right)^{\text {degree }(p)}\right)$ and for every natural number $i$ such that $i \in k$ holds $Y(i)>x+1$ and $1+(Z+1) \cdot(K !)=\prod(1+K ! \cdot(\operatorname{idseq}(x+1)))$ and $\left.\operatorname{eval}\left(p,{ }^{\circledR}((\langle Z, x\rangle\urcorner X)^{\wedge} Y\right)\right) \equiv$ $0(\bmod 1+(Z+1) \cdot(K !))$ and for every natural number $i$ such that $i \in k$ holds $\prod(Y(i)+1+-\operatorname{idseq}(x+1)) \equiv 0(\bmod 1+(Z+1) \cdot(K !))$.

Proof: Set $x_{1}=x+1$. If for every element $z$ of $\mathbb{N}$ such that $z \leqslant x$ there exists a $k$-element finite 0 -sequence $y$ of $\mathbb{N}$ such that for every $i$ such that $i \in k$ holds $y(i) \leqslant x$ and $\left.\operatorname{eval}\left(p,{ }^{\circledR}((\langle z, x\rangle\urcorner X) \frown y\right)\right)=0$, then there exists a $k$-element finite 0 -sequence $Y$ of $\mathbb{N}$ and there exist elements $Z, K$ of $\mathbb{N}$ such that $K>x$ and $K \geqslant\left(\sum \operatorname{coeff}(|p|)\right) \cdot\left(\left(x^{2}+1\right) \cdot\left(\prod(1+X)\right)^{\text {degree }(p)}\right)$ and for every natural number $i$ such that $i \in k$ holds $Y(i)>x_{1}$ and $1+(Z+1) \cdot(K !)=\prod(1+K ! \cdot(\operatorname{idseq}(x+1)))$ and $\left.\operatorname{eval}\left(p,{ }^{\circledR}((\langle Z, x\rangle\urcorner X)^{\frown} Y\right)\right) \equiv$ $0(\bmod 1+(Z+1) \cdot(K !))$ and for every natural number $i$ such that $i \in k$ holds $\prod\left(Y(i)+1+-\operatorname{idseq}\left(x_{1}\right)\right) \equiv 0(\bmod 1+(Z+1) \cdot(K !))$. Set $K_{1}=K !$. Set $z_{1}=1+(z+1) \cdot K_{1}$.

Consider $p_{3}$ being an element of $\mathbb{N}$ such that $p_{3} \mid z_{1}$ and $p_{3} \leqslant z_{1}$ and $p_{3}$ is prime. Define $\mathcal{P}($ object $)=Y\left(\$_{1}\right) \bmod p_{3}$. Consider $Y_{3}$ being a finite 0 -sequence such that len $Y_{3}=k$ and for every natural number $i$ such that $i \in k$ holds $Y_{3}(i)=\mathcal{P}(i) . \operatorname{rng} Y_{3} \subseteq \mathbb{N}$. Reconsider $E_{1}=\operatorname{eval}\left(p,{ }^{\circledR}\left(\left(\langle Z, x\rangle{ }^{\frown}\right.\right.\right.$ $\left.\left.X)^{\frown} Y\right)\right)$ as an integer. $K<p_{3}$. For every natural number $i$ such that $i \in k$ holds $Y_{3}(i) \leqslant x$. For every $i$ such that $i \in 2+k+n$ holds $p_{3} \mid\left(\left(\langle Z, x\rangle^{\frown} X\right)^{\frown}\right.$ $\left.Y)(i)-((\langle z, x\rangle\urcorner X)^{\frown} Y_{3}\right)(i) \cdot p_{3} \mid E_{1}-\operatorname{eval}\left(p,{ }^{\circledR}\left((\langle z, x\rangle \frown X) \frown Y_{3}\right)\right)$. Consider 
$m$ being a natural number such that $\left|\operatorname{eval}\left(p,{ }^{@}\left((\langle z, x\rangle \frown X) \frown Y_{3}\right)\right)\right|=p_{3} \cdot m$. For every object $i$ such that $i \in \operatorname{dom}\left({ }^{@}\left((\langle z, x\rangle \frown X) \frown Y_{3}\right)\right) \operatorname{holds} \mid\left({ }^{@}((\langle z, x\rangle \frown\right.$

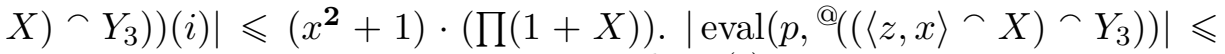
$\left(\sum \operatorname{coeff}(|p|)\right) \cdot\left(\left(x^{2}+1\right) \cdot\left(\prod(1+X)\right)^{\operatorname{degree}(p)}\right)$.

Let us consider a $\mathbb{Z}$ valued polynomial $p$ of $2+n+k, \mathbb{R}_{\mathrm{F}}$. Now we state the propositions:

(18) $\{X$, where $X$ is an $n$-element finite 0 -sequence of $\mathbb{N}$ : there exists an element $x$ of $\mathbb{N}$ such that for every element $z$ of $\mathbb{N}$ such that $z \leqslant x$ there exists a $k$-element finite 0 -sequence $y$ of $\mathbb{N} \operatorname{such}$ that $\operatorname{eval}\left(p,{ }^{@}\left((\langle z, x\rangle \frown X)^{\frown} y\right)\right)=$ $0\}$ is a Diophantine subset of the $n$-xtuples of $\mathbb{N}$.

Proof: Set $X_{0}=\{X$, where $X$ is an $n$-element finite 0 -sequence of $\mathbb{N}$ : there exists an element $x$ of $\mathbb{N}$ such that for every element $z$ of $\mathbb{N}$ such that $z \leqslant x$ there exists a $k$-element finite 0 -sequence $y$ of $\mathbb{N}$ such that $\left.\operatorname{eval}\left(p,{ }^{@}\left((\langle z, x\rangle \frown X)^{\frown} y\right)\right)=0\right\}$. Set $n_{1}=1+n+k$. Set $s_{4}=\sum \operatorname{coeff}(|p|)$. Set $D=\operatorname{degree}(p)$. Reconsider $Z_{0}=0, i_{0}=n_{1}, i_{1}=n_{1}+1, i_{2}=n_{1}+2$, $i_{3}=n_{1}+3$ as an element of $n_{1}+4$. Define $\mathcal{P}_{2}$ [finite 0 -sequence of $\left.\mathbb{N}\right] \equiv$ $1 \cdot \$_{1}\left(i_{1}\right)>1 \cdot \$_{1}\left(Z_{0}\right)+0$. Define $\mathcal{P}_{3}[$ finite 0 -sequence of $\mathbb{N}] \equiv \$_{1}\left(i_{1}\right) \geqslant$ $s_{4} \cdot\left(\left(\$_{1}\left(Z_{0}\right)^{2}+1\right) \cdot\left(\prod\left(1+\$_{1 \downarrow 1}\lceil n)\right) \cdot\left(1 \cdot \$_{1}\left(i_{0}\right)+0\right)^{0 \cdot \$_{1}\left(i_{0}\right)+D}\right) \cdot\{q\right.$, where $q$ is an $\left(n_{1}+4\right)$-element finite 0 -sequence of $\left.\mathbb{N}: \mathcal{P}_{3}[q]\right\}$ is a Diophantine subset of the $n_{1}+4$-xtuples of $\mathbb{N}$.

Define $\mathcal{P}_{4}[$ finite 0 -sequence of $\mathbb{N}] \equiv$ for every natural number $i$ such that $i \in k$ holds $\$_{1}(1+n+i)>\$_{1}\left(i_{0}\right)$ and $\prod\left(\$_{1}(1+n+i)+1+-\operatorname{idseq}\left(\$_{1}\left(i_{0}\right)\right)\right) \equiv$ $0\left(\bmod \$_{1}\left(i_{2}\right)\right)$. $\left\{q\right.$, where $q$ is an $\left(n_{1}+4\right)$-element finite 0 -sequence of $\mathbb{N}$ : $\left.\mathcal{P}_{4}[q]\right\}$ is a Diophantine subset of the $n_{1}+4$-xtuples of $\mathbb{N}$. Define $\mathcal{P}_{5}$ [finite 0 -sequence of $\mathbb{N}] \equiv 1 \cdot \$_{1}\left(i_{0}\right)>1 \cdot \$_{1}\left(Z_{0}\right)+0$. Define $\mathcal{P}_{6}$ [finite 0 -sequence of $\mathbb{N}] \equiv 1+\left(\$_{1}\left(i_{3}\right)+1\right) \cdot\left(\$_{1}\left(i_{1}\right) !\right)=\$_{1}\left(i_{2}\right)$. Define $\mathcal{P}_{7}[$ finite 0 -sequence of $\mathbb{N}] \equiv$ $\$_{1}\left(i_{2}\right)=\prod\left(1+\$_{1}\left(i_{1}\right)\right.$ ! $\cdot\left(\right.$ idseq $\left.\left.\left(1+\$_{1}\left(Z_{0}\right)\right)\right)\right)$. Reconsider $R=p$ as a $\mathbb{Z}$ valued polynomial of $1+n_{1}, \mathbb{R}_{F}$. Define $\mathcal{P}_{8}[$ finite 0 -sequence of $\mathbb{N}] \equiv$ for every $\left(1+n_{1}\right)$-element finite 0 -sequence $Y$ of $\mathbb{N}$ such that $Y=\left\langle \$_{1}\left(i_{3}\right)\right\rangle \frown\left(\$_{1}\left\lceil n_{1}\right)\right.$ $\operatorname{holds} \operatorname{eval}\left(R,{ }^{@} Y\right) \equiv 0\left(\bmod \$_{1}\left(i_{2}\right)\right) .\left\{q\right.$, where $q$ is an $\left(n_{1}+4\right)$-element finite 0 -sequence of $\left.\mathbb{N}: \mathcal{P}_{8}[q]\right\}$ is a Diophantine subset of the $n_{1}+4$-xtuples of $\mathbb{N}$.

Define $\mathcal{P}_{123}[$ finite 0 -sequence of $\mathbb{N}] \equiv \mathcal{P}_{2}\left[\$_{1}\right]$ and $\mathcal{P}_{3}\left[\$_{1}\right] .\{q$, where $q$ is an $\left(n_{1}+4\right)$-element finite 0 -sequence of $\left.\mathbb{N}: \mathcal{P}_{123}[q]\right\}$ is a Diophantine subset of the $n_{1}+4$-xtuples of $\mathbb{N}$. Define $\mathcal{P}_{1234}$ [finite 0 -sequence of $\mathbb{N}$ ] $\equiv$ $\mathcal{P}_{123}\left[\$_{1}\right]$ and $\mathcal{P}_{4}\left[\$_{1}\right] .\left\{q\right.$, where $q$ is an $\left(n_{1}+4\right)$-element finite 0 -sequence of $\left.\mathbb{N}: \mathcal{P}_{1234}[q]\right\}$ is a Diophantine subset of the $n_{1}+4$-xtuples of $\mathbb{N}$. Define $\mathcal{P}_{12345}[$ finite 0 -sequence of $\mathbb{N}] \equiv \mathcal{P}_{1234}\left[\$_{1}\right]$ and $\mathcal{P}_{5}\left[\$_{1}\right] .\left\{q\right.$, where $q$ is an $\left(n_{1}+\right.$ 4)-element finite 0 -sequence of $\left.\mathbb{N}: \mathcal{P}_{12345}[q]\right\}$ is a Diophantine subset of 
the $n_{1}+4$-xtuples of $\mathbb{N}$. Define $\mathcal{P}_{123456}$ [finite 0 -sequence of $\left.\mathbb{N}\right] \equiv \mathcal{P}_{12345}\left[\$_{1}\right]$ and $\mathcal{P}_{6}\left[\$_{1}\right]$. $\left\{q\right.$, where $q$ is an $\left(n_{1}+4\right)$-element finite 0 -sequence of $\mathbb{N}$ : $\left.\mathcal{P}_{123456}[q]\right\}$ is a Diophantine subset of the $n_{1}+4$-xtuples of $\mathbb{N}$. Define $\mathcal{P}_{1234567}[$ finite 0 -sequence of $\mathbb{N}] \equiv \mathcal{P}_{123456}\left[\$_{1}\right]$ and $\mathcal{P}_{7}\left[\$_{1}\right]$. $\{q$, where $q$ is an $\left(n_{1}+4\right)$-element finite 0 -sequence of $\left.\mathbb{N}: \mathcal{P}_{1234567}[q]\right\}$ is a Diophantine subset of the $n_{1}+4$-xtuples of $\mathbb{N}$.

Define $\mathcal{P}_{12345678}[$ finite 0 -sequence of $\mathbb{N}] \equiv \mathcal{P}_{1234567}\left[\$_{1}\right]$ and $\mathcal{P}_{8}\left[\$_{1}\right]$. Set $X_{3}=\left\{q\right.$, where $q$ is an $\left(n_{1}+4\right)$-element finite 0 -sequence of $\left.\mathbb{N}: \mathcal{P}_{12345678}[q]\right\}$. $X_{3}$ is a Diophantine subset of the $n_{1}+4$-xtuples of $\mathbb{N}$. Set $X_{2}=\{X \uparrow(n+$ $1)$, where $X$ is an $\left(n_{1}+4\right)$-element finite 0 -sequence of $\left.\mathbb{N}: X \in X_{3}\right\}$. Define $\mathcal{S}$ [finite 0 -sequence of $\mathbb{N}$ ] $\equiv$ for every element $z$ of $\mathbb{N}$ such that $z \leqslant \$_{1}(0)$ there exists a $k$-element finite 0 -sequence $y$ of $\mathbb{N}$ such that for every $n$-element finite 0 -sequence $X_{1}$ of $\mathbb{N}$ such that $X_{1}=\$_{1 \downarrow 1}$ holds $\operatorname{eval}\left(p,{ }^{\circledR}\left(\left(\left\langle z, \$_{1}(0)\right\rangle^{\frown} X_{1}\right)^{\frown} y\right)\right)=0$. Set $X_{1}=\{X$, where $X$ is an $(n+$ 1)-element finite 0 -sequence of $\mathbb{N}: \mathcal{S}[X]\}$. For every object $s, s \in X_{1}$ iff $s \in X_{2}$. Set $Y_{1}=\left\{X_{l 1}\right.$, where $X$ is an $(n+1)$-element finite 0 -sequence of $\left.\mathbb{N}: X \in X_{1}\right\}$. For every object $s, s \in Y_{1}$ iff $s \in X_{0}$.

(19) $\{X$, where $X$ is an $n$-element finite 0 -sequence of $\mathbb{N}$ : there exists an element $x$ of $\mathbb{N}$ such that for every element $z$ of $\mathbb{N}$ such that $z \leqslant x$ there exists a $k$-element finite 0 -sequence $y$ of $\mathbb{N}$ such that for every natural number $i$ such that $i \in k$ holds $y(i) \leqslant x$ and $\left.\operatorname{eval}\left(p,{ }^{\circledR}\left((\langle z, x\rangle \frown X)^{\frown} y\right)\right)=0\right\}$ is a Diophantine subset of the $n$-xtuples of $\mathbb{N}$.

Proof: Set $X_{0}=\{X$, where $X$ is an $n$-element finite 0 -sequence of $\mathbb{N}$ : there exists an element $x$ of $\mathbb{N}$ such that for every element $z$ of $\mathbb{N}$ such that $z \leqslant x$ there exists a $k$-element finite 0 -sequence $y$ of $\mathbb{N}$ such that for every natural number $i$ such that $i \in k$ holds $y(i) \leqslant x$ and $\operatorname{eval}(p$, $\left.\left.{ }^{\circledR}((\langle z, x\rangle \frown X) \frown y)\right)=0\right\}$. Set $n_{1}=1+n+k$. Set $s_{4}=\sum \operatorname{coeff}(|p|)$. Set $D=$ degree $(p)$. Reconsider $Z_{0}=0, i_{0}=n_{1}, i_{1}=n_{1}+1, i_{2}=n_{1}+2$, $i_{3}=n_{1}+3$ as an element of $n_{1}+4$. Define $\mathcal{P}_{2}$ [finite 0 -sequence of $\mathbb{N}$ ] $\equiv$ $1 \cdot \$_{1}\left(i_{1}\right)>1 \cdot \$_{1}\left(Z_{0}\right)+0$. Define $\mathcal{P}_{3}[$ finite 0 -sequence of $\mathbb{N}] \equiv \$_{1}\left(i_{1}\right) \geqslant$ $s_{4} \cdot\left(\left(\$_{1}\left(Z_{0}\right)^{2}+1\right) \cdot\left(\prod\left(1+\$_{1\lfloor 1}\lceil n)\right) \cdot\left(0 \cdot \$_{1}\left(i_{0}\right)+1\right)^{0 \cdot \$_{1}\left(i_{0}\right)+D}\right)\right.$. $\{q$, where $q$ is an $\left(n_{1}+4\right)$-element finite 0 -sequence of $\left.\mathbb{N}: \mathcal{P}_{3}[q]\right\}$ is a Diophantine subset of the $n_{1}+4$-xtuples of $\mathbb{N}$.

Define $\mathcal{P}_{4}$ [finite 0 -sequence of $\left.\mathbb{N}\right] \equiv$ for every natural number $i$ such that $i \in k$ holds $\$_{1}(1+n+i)>\$_{1}\left(i_{0}\right)$ and $\prod\left(\$_{1}(1+n+i)+1+-\operatorname{idseq}\left(\$_{1}\left(i_{0}\right)\right)\right) \equiv$ $0\left(\bmod \$_{1}\left(i_{2}\right)\right)$. $\left\{q\right.$, where $q$ is an $\left(n_{1}+4\right)$-element finite 0 -sequence of $\mathbb{N}$ : $\left.\mathcal{P}_{4}[q]\right\}$ is a Diophantine subset of the $n_{1}+4$-xtuples of $\mathbb{N}$. Define $\mathcal{P}_{5}$ [finite 0 -sequence of $\mathbb{N}$ ] $\equiv \$_{1}\left(i_{0}\right)=1 \cdot \$_{1}\left(Z_{0}\right)+1$. Define $\mathcal{P}_{6}$ [finite 0 -sequence of $\mathbb{N}] \equiv 1+\left(\$_{1}\left(i_{3}\right)+1\right) \cdot\left(\$_{1}\left(i_{1}\right) !\right)=\$_{1}\left(i_{2}\right)$. Define $\mathcal{P}_{7}[$ finite 0 -sequence of $\mathbb{N}] \equiv$ $\$_{1}\left(i_{2}\right)=\prod\left(1+\$_{1}\left(i_{1}\right) ! \cdot\left(\operatorname{idseq}\left(1+\$_{1}\left(Z_{0}\right)\right)\right)\right.$ ). Reconsider $R=p$ as a $\mathbb{Z}$ valued 
polynomial of $1+n_{1}, \mathbb{R}_{F}$. Define $\mathcal{P}_{8}$ [finite 0 -sequence of $\mathbb{N}$ ] $\equiv$ for every $\left(1+n_{1}\right)$-element finite 0 -sequence $Y$ of $\mathbb{N}$ such that $Y=\left\langle \$_{1}\left(i_{3}\right)\right\rangle \frown\left(\$_{1}\left\lceil n_{1}\right)\right.$ $\operatorname{holds} \operatorname{eval}\left(R,{ }^{\circledR} Y\right) \equiv 0\left(\bmod \$_{1}\left(i_{2}\right)\right) .\left\{q\right.$, where $q$ is an $\left(n_{1}+4\right)$-element finite 0 -sequence of $\left.\mathbb{N}: \mathcal{P}_{8}[q]\right\}$ is a Diophantine subset of the $n_{1}+4$-xtuples of $\mathbb{N}$.

Define $\mathcal{P}_{123}[$ finite 0 -sequence of $\mathbb{N}] \equiv \mathcal{P}_{2}\left[\$_{1}\right]$ and $\mathcal{P}_{3}\left[\$_{1}\right] .\{q$, where $q$ is an $\left(n_{1}+4\right)$-element finite 0 -sequence of $\left.\mathbb{N}: \mathcal{P}_{123}[q]\right\}$ is a Diophantine subset of the $n_{1}+4$-xtuples of $\mathbb{N}$. Define $\mathcal{P}_{1234}$ [finite 0 -sequence of $\mathbb{N}$ ] $\equiv$ $\mathcal{P}_{123}\left[\$_{1}\right]$ and $\mathcal{P}_{4}\left[\$_{1}\right] .\left\{q\right.$, where $q$ is an $\left(n_{1}+4\right)$-element finite 0 -sequence of $\left.\mathbb{N}: \mathcal{P}_{1234}[q]\right\}$ is a Diophantine subset of the $n_{1}+4$-xtuples of $\mathbb{N}$. Define $\mathcal{P}_{12345}[$ finite 0 -sequence of $\mathbb{N}] \equiv \mathcal{P}_{1234}\left[\$_{1}\right]$ and $\mathcal{P}_{5}\left[\$_{1}\right] .\left\{q\right.$, where $q$ is an $\left(n_{1}+\right.$ 4)-element finite 0 -sequence of $\left.\mathbb{N}: \mathcal{P}_{12345}[q]\right\}$ is a Diophantine subset of the $n_{1}+4$-xtuples of $\mathbb{N}$. Define $\mathcal{P}_{123456}$ [finite 0 -sequence of $\left.\mathbb{N}\right] \equiv \mathcal{P}_{12345}\left[\$_{1}\right]$ and $\mathcal{P}_{6}\left[\$_{1}\right] .\left\{q\right.$, where $q$ is an $\left(n_{1}+4\right)$-element finite 0 -sequence of $\mathbb{N}$ : $\left.\mathcal{P}_{123456}[q]\right\}$ is a Diophantine subset of the $n_{1}+4$-xtuples of $\mathbb{N}$. Define $\mathcal{P}_{1234567}[$ finite 0 -sequence of $\mathbb{N}] \equiv \mathcal{P}_{123456}\left[\$_{1}\right]$ and $\mathcal{P}_{7}\left[\$_{1}\right] .\{q$, where $q$ is an $\left(n_{1}+4\right)$-element finite 0 -sequence of $\left.\mathbb{N}: \mathcal{P}_{1234567}[q]\right\}$ is a Diophantine subset of the $n_{1}+4$-xtuples of $\mathbb{N}$. Define $\mathcal{P}_{12345678}[$ finite 0 -sequence of $\mathbb{N}$ ] $\equiv$ $\mathcal{P}_{1234567}\left[\$_{1}\right]$ and $\mathcal{P}_{8}\left[\$_{1}\right]$. Set $X_{3}=\left\{q\right.$, where $q$ is an $\left(n_{1}+4\right)$-element finite 0 -sequence of $\left.\mathbb{N}: \mathcal{P}_{12345678}[q]\right\} . X_{3}$ is a Diophantine subset of the $n_{1}+4$ xtuples of $\mathbb{N}$. Set $X_{2}=\left\{X \uparrow(n+1)\right.$, where $X$ is an $\left(n_{1}+4\right)$-element finite 0-sequence of $\left.\mathbb{N}: X \in X_{3}\right\}$.

Define $\mathcal{S}$ [finite 0 -sequence of $\mathbb{N}] \equiv$ for every element $z$ of $\mathbb{N}$ such that $z \leqslant \$_{1}(0)$ there exists a $k$-element finite 0 -sequence $y$ of $\mathbb{N}$ such that for every $n$-element finite 0 -sequence $X_{1}$ of $\mathbb{N}$ such that $X_{1}=\$_{1 \downarrow 1}$ holds for every $i$ such that $i \in k$ holds $y(i) \leqslant \$_{1}(0)$ and $\operatorname{eval}\left(p,{ }^{@}\left(\left(\left\langle z, \$_{1}(0)\right\rangle \frown X_{1}\right)^{\frown}\right.\right.$ $y))=0$. Set $X_{1}=\{X$, where $X$ is an $(n+1)$-element finite 0-sequence of $\mathbb{N}: \mathcal{S}[X]\}$. For every object $s, s \in X_{1}$ iff $s \in X_{2}$. Set $Y_{1}=\left\{X_{11}\right.$, where $X$ is an $(n+1)$-element finite 0 -sequence of $\left.\mathbb{N}: X \in X_{1}\right\}$. For every object $s, s \in Y_{1}$ iff $s \in X_{0}$.

Let $n$ be a natural number and $A$ be a subset of the $n$-xtuples of $\mathbb{N}$. We say that $A$ is recursively enumerable if and only if

(Def. 4) there exists a natural number $m$ and there exists a $\mathbb{Z}$ valued polynomial $P$ of $2+n+m, \mathbb{R}_{\mathrm{F}}$ such that for every $n$-element finite 0 -sequence $X$ of $\mathbb{N}, X \in A$ iff there exists an element $x$ of $\mathbb{N}$ such that for every element $z$ of $\mathbb{N}$ such that $z \leqslant x$ there exists an $m$-element finite 0 -sequence $Y$ of $\mathbb{N}$ such that for every object $i$ such that $i \in \operatorname{dom} Y$ holds $Y(i) \leqslant x$ and $\operatorname{eval}\left(P,{ }^{@}((\langle z, x\rangle \frown X) \frown Y)\right)=0$.

Now we state the proposition: 
(20) Let us consider a natural number $n$, and a subset $A$ of the $n$-xtuples of $\mathbb{N}$. If $A$ is Diophantine, then $A$ is recursively enumerable.

Proof: Consider $m$ being a natural number, $P$ being a $\mathbb{Z}$ valued polynomial of $n+m, \mathbb{R}_{\mathrm{F}}$ such that for every object $s, s \in A$ iff there exists an $n$-element finite 0 -sequence $x$ of $\mathbb{N}$ and there exists an $m$-element finite 0 -sequence $y$ of $\mathbb{N}$ such that $s=x$ and $\operatorname{eval}\left(P,{ }^{\circledR}(x \frown y)\right)=0$. Set $n_{4}=n+m$. Reconsider $\mathrm{P}_{0}=P$ as a $\mathbb{Z}$ valued polynomial of $0+n_{4}, \mathbb{R}_{\mathrm{F}}$. Consider $q$ being a polynomial of $0+2+n_{4}, \mathbb{R}_{\mathrm{F}}$ such that $\operatorname{rng} q \subseteq \operatorname{rng} \mathrm{P}_{0} \cup\left\{0_{\mathbb{R}_{F}}\right\}$ and for every function $x_{1}$ from $0+n_{4}$ into $\mathbb{R}_{\mathrm{F}}$ and for every function $X_{1}$ from $0+2+n_{4}$ into $\mathbb{R}_{\mathrm{F}}$ such that $x_{1} \uparrow 0=X_{1} \uparrow 0$ and $\left({ }^{@} x_{1}\right)_{\downarrow 0}=\left({ }^{@} X_{1}\right)_{\downarrow} 0+2$ holds $\operatorname{eval}\left(\mathrm{P}_{0}, x_{1}\right)=\operatorname{eval}\left(q, X_{1}\right)$.

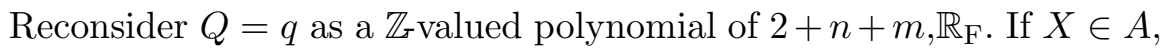
then there exists an element $x$ of $\mathbb{N}$ such that for every element $z$ of $\mathbb{N}$ such that $z \leqslant x$ there exists an $m$-element finite 0 -sequence $Y$ of $\mathbb{N}$ such that for every object $i$ such that $i \in \operatorname{dom} Y$ holds $Y(i) \leqslant x$ and $\operatorname{eval}\left(Q,{ }^{@}((\langle z, x\rangle \frown X) \frown Y)\right)=0$. Consider $y$ being an $m$-element finite 0 -sequence of $\mathbb{N}$ such that for every object $i$ such that $i \in \operatorname{dom} y$ holds $y(i) \leqslant a$ and $\operatorname{eval}\left(Q,{ }^{\circledR}((\langle a, a\rangle \frown X) \frown y)\right)=0$.

\section{MRDP THEOREM}

Now we state the proposition:

(21) Yuri Matiyasevich, Julia Robinson, Martin Davis, Hilary PutNAM THEOREM:

Let us consider a natural number $n$, and a subset $A$ of the $n$-xtuples of $\mathbb{N}$. If $A$ is recursively enumerable, then $A$ is Diophantine. The theorem is a consequence of (19).

\section{REFERENCES}

[1] Marcin Acewicz and Karol Pak. Basic Diophantine relations. Formalized Mathematics, 26(2):175-181, 2018. doi 10.2478/forma-2018-0015.

[2] Marcin Acewicz and Karol Pak. Pell's equation. Formalized Mathematics, 25(3):197-204, 2017. doi $10.1515 /$ forma-2017-0019.

[3] Zofia Adamowicz and Paweł Zbierski. Logic of Mathematics: A Modern Course of Classical Logic. Pure and Applied Mathematics: A Wiley Series of Texts, Monographs and Tracts. Wiley-Interscience, 1997.

[4] Grzegorz Bancerek, Czesław Byliński, Adam Grabowski, Artur Korniłowicz, Roman Matuszewski, Adam Naumowicz, Karol Pąk, and Josef Urban. Mizar: State-of-the-art and beyond In Manfred Kerber, Jacques Carette, Cezary Kaliszyk, Florian Rabe, and Volker Sorge, editors, Intelligent Computer Mathematics, volume 9150 of Lecture Notes in Computer Science, pages 261-279. Springer International Publishing, 2015. ISBN 978-3319-20614-1. doi $10.1007 / 978-3-319-20615-8 \_17$. 
[5] Grzegorz Bancerek, Czesław Byliński, Adam Grabowski, Artur Korniłowicz, Roman Matuszewski, Adam Naumowicz, and Karol Pak. The role of the Mizar Mathematical Library for interactive proof development in Mizar. Journal of Automated Reasoning, 61(1):9-32, 2018. do1 $10.1007 /$ s10817-017-9440-6

[6] Martin Davis. Hilbert's tenth problem is unsolvable. The American Mathematical Monthly, Mathematical Association of America, 80(3):233-269, 1973. doi $10.2307 / 2318447$.

[7] Adam Grabowski, Artur Korniłowicz, and Adam Naumowicz. Four decades of Mizar. Journal of Automated Reasoning, 55(3):191-198, 2015. doi:10.1007/s10817-015-9345-1

[8] Karol Pąk. The Mativasevich theorem. Preliminaries. Formalized Mathematics, 25(4): 315-322, 2017. doi $10.1515 /$ forma-2017-0029

[9] Karol Pak. Diophantine sets. Part II. Formalized Mathematics, 27(2):197-208, 2019. doi: 10.2478/forma-2019-0019.

[10] Craig Alan Smorynski. Logical Number Theory I, An Introduction. Universitext. SpringerVerlag Berlin Heidelberg, 1991. ISBN 978-3-642-75462-3.

Accepted May 27, 2019 